\title{
Space-based visible observation strategy for beyond-LEO objects based on an equatorial LEO satellite with multi-sensors
}

\author{
Yun-peng $\mathrm{Hu}^{\mathrm{a}}$, Jian-yu Huang ${ }^{\mathrm{b}}$, Lei Chen ${ }^{\mathrm{a}, *}$ \\ ${ }^{a}$ College of Aerospace Science and Engineering, National University of Defense Technology, \\ Changsha, 410073 \\ ${ }^{b}$ Beijing Institute of Tracking and Telecommunication Technology, Beijing, 100094
}

Yun-peng Hu. Mailing addresses: Deya road no. 109, Kaifu district, Changsha, Hunan Province, China. Email address: huyunpengnudt@163.com.

Jian-yu Huang. Mailing addresses: Beiqing road no. 26, Haidian district, Beijing, China. Email address: jianyu2875@126.com.

Lei Chen. Mailing addresses: Deya road no. 109, Kaifu district, Changsha, Hunan Province, China. Email address: chenl@nudt.edu.cn.

* Corresponding author. Tel.: +86 0731-87001385; fax: +86 0731-87001385. Email addresses: chenl@nudt.edu.cn (L. Chen). 


\title{
Space-based visible observation strategy for beyond-LEO objects
}

\section{based on an equatorial LEO satellite with multi-sensors}

\author{
Yun-peng $\mathrm{Hu}^{\mathrm{a}}$, Jian-yu Huang ${ }^{\mathrm{b}}$, Lei Chen ${ }^{\mathrm{a}, *}$ \\ ${ }^{a}$ College of Aerospace Science and Engineering, National University of Defense Technology, \\ Changsha, 410073 \\ ${ }^{b}$ Beijing Institute of Tracking and Telecommunication Technology, Beijing, 100094
}

\begin{abstract}
Many space-based visible observation strategies based on Low Earth Orbit (LEO) satellites for observing Geosynchronous Orbit (GEO) objects were proposed previously. However, there were few studies about other beyond-LEO objects (Geostationary Transfer Orbit (GTO) objects, Medium Earth Orbit (MEO) objects, and Molniya objects). In this paper, a space-based visible observation strategy is proposed for observing GEO objects, GTO objects, MEO objects (especially global navigation satellites), and Molniya objects simultaneously to get more orbital data, using an earth-oriented equatorial LEO satellite with three sensors. This work is focused on the pointing geometry. Brightness of observed objects and sensitivity of sensors are assumed under the relative ideal conditions. First, the distribution characteristics of these beyond-LEO objects are discussed. And in order to observe global navigation satellites efficiently, joint regions formed by the track superposition of two adjacent orbits in a constellation are proposed. To offset the influence of the earth shadow and constraint of sun-target-observer angle, two sensors pointing inside of the equatorial plane are used to observe GEO and GTO objects. The installation angle of the third sensor is optimized to obtain a relative high coverage rate for observing global navigation satellites and Molniya objects based on joint regions. Finally, the coverage rate, the number of observations, and observation duration under different sensors with different field of views (FOVs) are compared and analyzed respectively.
\end{abstract}

Keywords: Space-based visible observation; Equatorial LEO satellite; Multi- sensors; Joint regions

\section{Introduction}

Some kinds of beyond-LEO are widely used for aerospace activities because of their important application values. Due to its specific orbital characteristics, GEO is an important and limited strategic resource; Molniya orbit is a kind of high elliptical orbit, which is often used for navigation, communication, and scientific observation near the Arctic region (Alexander and Louis, 2011); MEO objects mainly consist of global navigation satellites constellations. An increasing number of global navigation satellites are launched into MEO when more and more countries have recognized the importance of global navigation satellites system (GNSS) (Christopher, 2008). Most of them belong to Global Positing System (GPS) operated by the United States (U.S.), which consists of six equiphase orbital planes inclined about $55^{\circ}$ with an orbital radius of about 26559km (Kaplan and Hegarty, 2006) and the GLONASS satellite navigation system operated by the Russian Federation, which consists of three orbital planes, with an inclination of about $64.8^{\circ}$ and an orbital radius of about $25378 \mathrm{~km}$ (Revnivykh, 2007). Others are mainly Galileo navigation satellites operated by European Union

${ }^{*}$ Corresponding author. Tel.: +86 0731-87001385; fax: +86 0731-87001385. Email addresses: chenl@nudt.edu.cn (L. Chen). 
(Zandbergen et al., 2004) and some of the BeiDou/Compass Navigation Test System (BNTS) satellites operated by China (Xiaoli et al., 2013). Most inclinations and orbital radius of global navigation satellites are in the region of $\left(52^{\circ}, 68^{\circ}\right)$ and $(24000 \mathrm{~km}, 28000 \mathrm{~km})$, respectively.

With the number of GEO objects steadily increasing over time, GEO objects will suffer more chances of the collision threat from GEO and GTO objects (Klinkrad, 2003). And with the increasing of global navigation satellites, the problem of their safety is becoming more and more severe. Thus, one way to guarantee the safety of these objects is to observe them effectively and timely so that they can do avoidance maneuvers if necessary.

After the U.S. Lincoln Laboratory proposing the space-based visible observation for space objects (Stokes et al., 1998), observing High Earth Orbit objects, especially GEO objects by a LEO satellite was proved to be feasible and efficient, so that LEO satellites are widely used for space-based visible observation of important space objects. Previously, many strategies were proposed for observing GEO objects based on sun-synchronous orbit satellites. The U.S. (Stokes et al., 1998; Sharma, 2000) and Canada (Maskell and Oram, 2008; Scott et al., 2013) had launched space-based observation into actual use. Meanwhile, ESA also proposed the passive and active observation mode of space-based observation for GEO objects, which achieved a fine efficiency (Flohrer et al., 2011; Utzmann et al., 2014; Silha et al., 2014).

However, it is difficult to observe all the GEO objects in one day and the revisit frequency is usually low in a relative short time using a sun-synchronous orbit satellite. Therefore, the strategy of using an equatorial LEO satellite, which can revisit GEO objects multiple times in a day (Sánchez et al., 2008; Utzmann and Wagner, 2011) was proposed. And the U.S. will launch the ORS-5 satellite into the equatorial LEO with $625 \mathrm{~km}$ altitude in the late spring of 2017, in order to observe GEO belt uninterruptedly by a flight geometry and sensor arrangement (Davis, et al., 2016). But there are lack of analysis and modification for this strategy.

Moreover, most of the observing strategies are mainly for GEO objects, while few strategies for observing other beyond-LEO objects, such as GTO, MEO, and Molniya objects are proposed. Previously, solutions for observing GEO and MEO objects were based on ground-based facilities, and a nearly complete coverage for these regions were obtained under the proposed space surveillance system (Flohrer, et al., 2008). On the aspect of space-based solutions, the strategies of using a constellation, which consist of multiple sun-synchronous satellites, or multiple equatorial LEO satellites, or both of them, were proposed and optimized to observe beyond-LEO objects (Oswald, et al. 2009).

In this paper, a strategy of general observation for simultaneously observing GEO, GTO, MEO and Molniya objects is proposed to get more data of these important objects using an earth-oriented equatorial LEO satellite with three sensors, as it is illustrated in Fig. 1. And it assumes that objects can be observed effectively, if their sun-target-observer angles are less than $150^{\circ}$. Other influence factors, such as brightness properties of observed objects and sensitivity of sensors, are not considered in this paper. Distribution characteristics of these objects are discussed in section 2. The operation characteristics for an earth-oriented equatorial LEO satellite are illustrated and the pointing strategies of sensors for different objects are designed respectively in section 3. In order to observe more global navigation satellites, the pointing of the sensor is optimized in section 4 . The detailed observation results for GEO, GTO, MEO and Molniya objects are performed and discussed in section 5 .

2. Distribution characteristics of beyond-LEO objects 


\subsection{Characteristics of GEO and GTO objects}

Ideal GEO is a circle orbit inclined $0^{\circ}$. However, the inclination $i$, eccentricity $e$, and semi-major axis $R$ of a practical GEO have small deviation $i, e$, and $\Delta R$, which can cause the drifting motion of a GEO object relative to its fix point in the earth. The inclination deviation is the primary reason which causes the change of the object's track of sub-satellite point in the direction of latitude. If only considering the factor of inclination deviation, the longitudinal and latitudinal deviation $\Delta \lambda, \Delta \varphi$ relative to the fixed point in the earth can be expressed as (Hengnian, 2010)

$$
\left\{\begin{array}{l}
\Delta \lambda=-\frac{i^{2}}{4} \sin \left(2 \omega_{e} t\right) \\
\Delta \varphi=i \cdot \sin \left(\omega_{e} t\right)
\end{array}\right.
$$

where $\omega_{e}$ is the rotational angular velocity of the earth, and $t$ is the time relative to the moment when the object passing through its ascending node firstly.

On the other hand, inclinations of GEO objects are generally less than $15^{\circ}$. GEO objects can be divided into two kinds. One is GEO objects under control, whose inclinations always keep near $0^{\circ}$. Another one is GEO objects beyond control, whose inclinations exist in the region of about $\left(0^{\circ}, 15^{\circ}\right)$ with an evolvement period of about 54 years (Soop, 1994). Moreover, the right ascension of ascending nodes (RAANs) of GEO objects beyond control are almost in the region of $\left(-90^{\circ}, 90^{\circ}\right)$ (Sharma et al., 2002). Meanwhile, GEO belt has the equal-widths and sinusoidal shapes in the inertial space (Yunpeng et al., 2016). And centers of GEO belt are $0^{\circ}$ declinations both at vernal equinox and autumnal equinox, while the centers of GEO belt are about $7.5^{\circ}$ and $-7.5^{\circ}$ declinations at summer solstice and winter solstice respectively. The key of the space-based visible strategy for observing GEO objects efficiently is whether the whole GEO belt could be covered within a relative short time or not.

GTO objects have large eccentricities. Their apogees are in the GEO belt, while their perigees are near the surface of the earth. So, GTO objects have relative slow velocities near their apogees. And the strategy for observing GTO objects is to observe them when they pass through their apogees.

Therefore, it is necessary to utilize a LEO satellite scan the field of sky near the declination $0^{\circ}$ for observing GEO and GTO objects simultaneously according to their distribution characteristics.

\subsection{Joint regions of MEO objects}

MEO objects mainly consist of global navigation satellites constellations. Global navigation satellites of one constellation have the similar orbital parameters. The main characteristic for different global navigation constellations is that satellites of the same constellation are distributed in the orbits with equal phase in the inertial space. Thus, trajectories of satellites in the adjacent two orbits of the same constellation have a superposition on the celestial sphere. The ideal superposition of the satellites' trajectories is called joint point such as the point $A$ in Fig. 2. Namely, all the objects in the two adjacent orbits will pass through the point and the possibility of the objects appearing at the point is the largest. Therefore, it is efficient to scan these joint points for observing global navigation satellites.

Assuming that two adjacent orbits of the same constellation have the same inclination $i$, and the difference between their RAANs is $\Delta \Omega$. The geometrical relationship between the two orbits is illustrated by the spherical triangle $A B C$ in Fig. 2, where $a=\Delta \Omega, B=i$, and $C=180^{\circ}-i$. According to spherical trigonometry, we have 


$$
\cos A=-\cos i \cos \left(180^{\circ}-i\right)+\sin i \sin \left(180^{\circ}-i\right) \cos \Delta \Omega
$$

Namely

$$
A=\arccos \left(\cos ^{2} i+\sin ^{2} i \cos a\right)
$$

Also according to spherical trigonometry, yields

$$
\sin c=\frac{\sin a}{\sin A} \sin C
$$

Meanwhile, in the right spherical triangle $A B C$ ', we have

$$
\sin b^{\prime}=\sin c \sin B
$$

where $b^{\prime}$ is the latitude $\delta$ of the joint point $A$. Eliminating Eqs. (2), (3), and (5) into Eq. (4), leads to

$$
\delta=\arcsin \left(\frac{\sin \Delta \Omega \sin ^{2} i}{\sin \left(\arccos \left(\cos ^{2} i+\sin ^{2} i \cos \Delta \Omega\right)\right)}\right)
$$

Because of the characteristic of the equiphase distribution for multiple orbits of the same constellation, there are multiple joint points distributed with circular array on the celestial sphere. The line $A D$ in Fig. 2 is the radius $r$ of the altitude circle of joint points. According to their geometrical relationship, $r$ and altitudes of joint points relative to the equatorial plane $h$ are

$$
\left\{\begin{array}{l}
r=R \cos \delta \\
h=R \sin \delta
\end{array}\right.
$$

where $R$ is the semi-major axis of global navigation satellites.

Taking GPS and GLONASS constellation for examples, the theoretical joint points of the two constellations are illustrated in Fig. 3 and Fig. 4 respectively. In practice, trajectories of satellites in the adjacent two orbits would not intersect on a point any more for the little difference of satellites' orbits, but form intersection areas which are called joint regions. The practical joint regions mainly formed by GPS satellites whose inclinations are in the region of $\left(52^{\circ}, 60^{\circ}\right)$, and GLONASS satellites whose inclinations are in the region of $\left(60^{\circ}, 68^{\circ}\right)$, are illustrated in Fig. 5(a) and Fig. 5(b) respectively. Although satellites are acted by perturbation forces, their orbit parameters of the same constellation are similar, relative position of them will not change obviously in the future, so do $r$ and $h$ of joint regions. Circles in Fig. 5 refer to the altitude circles of joint points. All the joint regions distribute near the circumferences. Analogously, there also exist joint regions distributed symmetrically in the south. Therefore, it is a feasible way to observe global navigation satellites by observing these joint regions.

\subsection{Distribution of Molniya objects}

Molniya is a type of highly elliptical orbit with an inclination of about $63.4^{\circ}$ and an orbital period of one half of a sidereal day. Its apogee is always kept in the north, and argument of perigee is about $270^{\circ}$. Therefore, Molniya satellite costs most time within an orbital period to pass through its apogee. Unlike global navigation satellites, Molniya objects have no obvious distribution regularity. Their RAANs exist in the whole range of $\left(-180^{\circ}, 180^{\circ}\right)$. But their apogees are distributed with a circular array in the celestial sphere as well for their similar orbital parameters except RAANs.

The practical distribution of Molniya objects' trajectories near their apogees is illustrated in Fig. 6. Their apogees are distributed around the circumference with the radius of about $20000 \mathrm{~km}$, and their trajectories are inside of this altitude circle. So, it is a feasible way to observe Molniya objects by 
covering regions near their apogees using a satellite with a short orbital period.

\subsection{Summary}

Radiuses of altitude circles, altitudes to equatorial plane, and latitudes of joint regions of GPS, GLONASS and Molniya objects are approximately listed in Table 1. Joint regions of these three kinds of objects are in the different fields of sky. Therefore, it is necessary that observation regions of the sensor cover these fields of sky to observe these objects.

\section{Strategy for observing beyond-LEO objects}

An equatorial LEO satellite has a short orbital period, and can operate multiple rounds in a day. Observation region of the sensor can cover some specific fields of sky with specific declinations, if the satellite is earth-oriented and the sensor is fixed on the satellite with a given pointing.

Orbits of equatorial LEO satellites are approximately coplanar with GEO and GTO. The whole GEO belt could be covered by utilizing the characteristics of equatorial LEO satellites, if the pointing of the sensor is inside of the equatorial plane. And if the sensor points outside of the equatorial plane, some specific fields with specific declinations could be covered as well. In order to observe different fields of sky, equatorial LEO satellites should be used and the installation angle of the sensor which is between the pointing of sensor and the orbital plane should be adjusted. According to section 2, those objects are distributed in different fields of sky with different declinations. Therefore, an earth-oriented equatorial LEO satellite is selected in this paper so that these objects can be observed simultaneously.

\subsection{Strategy for observing GEO and GTO objects}

The disadvantage for observing GTO objects using a sun-synchronous orbit satellite is that the observation region of the sensor fixed on a sun-synchronous orbit satellite is almost unchanged within a relative short time in the inertial space, and GTO objects whose apogees don't exist within the observation region could not be observed. However, a sensor fixed on an equatorial LEO satellite has a broad scanning region. All the objects in the whole GEO region can be revisited multiple times in a day in theory. Meanwhile, all the GTO objects have possibilities to be observed multiple times in a day as well. Taking observing GEO objects as the example, the strategy is illustrated as follows.

The effective observation region will reduce if the orbit altitude rises and the FOV of the sensor is a constant, which is illustrated in Fig. 7. Assuming that the pointing of the sensor is along the direction of the satellite's position vector in the Earth Centered Inertial System (ECI), and the FOV of the sensor is $P^{\circ} \times Q^{\circ}$, while the effective FOV is $p^{\circ} \times q^{\circ}$ when the observation region project to the GEO belt or the earth. According to Fig. 7, we have

$$
\left\{\begin{array}{l}
\tan \frac{p}{2}=\frac{A C}{O C} \\
\tan \frac{P}{2}=\frac{A C}{D C} \\
D C=O C-O D=O C-r \\
O C=O A \cos \frac{p}{2}=R_{G E O} \cos \frac{p}{2}
\end{array}\right.
$$

where $r$ is the radius of the equatorial satellite and $R_{G E O}$ is the radius of GEO belt. So 


$$
\tan \frac{P}{2}=\frac{R_{G E O} \cos (p / 2)}{R_{G E O} \cos (p / 2)-r} \tan \frac{p}{2}
$$

Considering $p / 2$ and $P / 2$ are small angles, yield to

$$
P \approx \frac{R}{R-r} p
$$

It is similar for the relationship between $Q$ and $q$

$$
Q \approx \frac{R}{R-r} q
$$

According to Eqs. (1) and (9), a GEO object can be revisited once within every orbital period of the observation satellite if $\Delta \varphi \leq p$, while a GEO object can only be observed when it passes through its ascending node and descending node if $\Delta \varphi>p$. These cases are illustrated in Fig. 8. According to Eq. (9), if the altitude of the observation satellite rises, the effective observation region will decline, and the FOV is required to be boarder especially in the direction of latitude to cover the whole GEO belt.

The observation duration for GEO objects using an equatorial LEO satellite is mainly determined by the orbital period of the observation satellite and the FOV in the direction of longitude $Q$. While the number of observations for GEO objects is mainly determined by the orbital period of the observation satellite and the FOV in the direction of latitude $P$. In the most cases, GTO objects will be observed when they pass through their apogees, so that the observation strategy is efficient for GTO objects if it is efficient for observing GEO objects.

In practice, observation of GEO and GTO objects is restrained by the sun-target-observer angle. The optical observation condition is bad if the phase angle is too large. As a result, the effective observation region is not the whole GEO belt any more, but has a small blind zone, which is illustrated in Fig. 8. Meanwhile, because of the influence of the shelter of earth shadow near the vernal equinox and autumnal equinox, the effective observation region will reduce about $23.5^{\circ}$ (Flohrer et al., 2011) during spring and autumn. So the number of observations in practice for observing GEO and GTO objects is slightly less than that in theory.

In order to improve observation capabilities in a day and offset the influence of the sun-target-observer angle and the earth shadow, the strategy of a satellite using two sensors to observe GEO and GTO objects is proposed. The sun-target-observer angle is relative to the pointing of the sensor. Assuming that acceptable sun-target-observer angle is $\alpha$, the effective observation of GEO belt is $2 \alpha$. The two sensors point inside of the equatorial plane, one of them is along the direction of its position vector in ECI, and another pointing has a deflection angle $\Delta$ in the direction of longitude with the first one. Assuming the sunlight is parallel to the equatorial plane, the effective observation regions of the two sensors are illustrated in Fig. 9(a). Deflection angle $\Delta$ has changed the effective observation region. And the two sensors' observation regions are complementary with each other. Therefore, the blind zone restrained by the sun-target-observer angle will reduce.

The optical observation condition is influenced by many factors, such as surface materials and shapes, flying conditions and working states (Yi et al., 2014). In this paper, considering that objects can be effectively observed if the sun-target-observer angle is less than $150^{\circ}$. The practical effective observation region in a spring day for GEO objects are illustrated in Fig. 9(b). One of the sensors points along the direction of its position vector in ECI, and another is along with the direction of its velocity vector, namely $\Delta=90^{\circ}$. The orbital period of the observation satellite is 13.5 revolutions per day. The gap at the top of the region is caused by the influence of the earth shadow, and the gap at the bottom is restrained by the sun-target-observer angle. It reveals that one of the observation regions of a sensor offsets a part of another one.

\subsection{Strategy for observing MEO and Molniya objects}

According to section 2, Global navigation satellites can be observed efficiently if the observation region covers their joint regions. With the characteristics of earth-oriented equatorial LEO satellites, the 
whole joint regions of global navigation satellites could be covered by the sensor fixed on the satellite with an appointed pointing. According to the position of joint regions, the sensor can point to the north or the south. Assuming that the sensor points to the joint regions in the south sky, the observation for global navigation satellites is illustrated in Fig. 10 and we have

$$
O B \cdot \tan \delta=(O B-O A) \cdot \tan \Phi
$$

where $O B=r, O A=R$, and $\Phi$ is the deflection angle of the pointing of the sensor in the direction of latitude. Substituting Eq. (7) into Eq. (11) yields

$$
\Phi=\tan ^{-1}\left(\frac{\tan \delta}{1-\tan \delta}\right)
$$

If the obliquity of the sunlight is $\varepsilon$, which has a variation period of one year, sun-target-observer angle in an observation period will change from $\Phi-\varepsilon$ to $180^{\circ}-\Phi-\varepsilon$. During the vernal equinox and the autumnal equinox, $\varepsilon$ is about $0^{\circ}$, and the sun light is parallel to the equatorial plane. The minimum of the sun-target-observer angle is $\Phi$, and the maximum is $180^{\circ}-\Phi$, when the satellite passes through between the earth and sun. While during the summer solstice and the winter solstice, $\varepsilon$ reaches the extremes of about $23.5^{\circ}$ and $-23.5^{\circ}$, and the variation ranges of sun-target-observer angles are $\left(\Phi-23.5^{\circ}, 180^{\circ}-\Phi-23.5^{\circ}\right)$, and $\left(\Phi+23.5^{\circ}, 180^{\circ}-\Phi+23.5^{\circ}\right)$ respectively. In the whole year, the sun-target-observer angle will not exceed the maximum of $180^{\circ}-\Phi+23.5^{\circ}$ which is always less than $180^{\circ}$. So it wouldn't cause a very bad optical observation condition in the whole observation period using equatorial LEO satellite.

According to Eq. (12), $\Phi$ should be adjusted about $66.3^{\circ}$ to cover the joint regions formed by GPS constellation satellites. And to cover the joint regions formed by GLONASS constellation satellites, $\Phi$ should be adjusted about $61.6^{\circ}$. Analogously, for observing Molniya objects near their apogees, the sensor should point to the north and $\Phi$ should be adjusted about $60.8^{\circ}$. As a result, although joint regions of these three kinds of objects differ sharply in inertial space, the required deflection angles $\Phi$ for observing them are similar. Therefore, if the sensor points to the north with an appropriate angle $\Phi$, and has a board FOV especially in the direction of latitude, global navigation satellites and Molniya objects can be observed effectively and simultaneously.

\section{Optimizing for the pointing of the sensor to observe MEO objects}

According to the characteristics of GEO and GTO objects, the observation results will be fine if the pointing of sensor point inside of the equatorial plane, so that it doesn't need to be optimized. Meanwhile, Molniya objects have relatively slow velocities near their apogees. They can be observed easily in the north sky. However, joint regions are generally ranges. It is necessary to optimize the pointing of the sensor for MEO objects.

Joint regions are formed by different global navigation constellations. Deflection angle of the pointing of the sensor in the direction of latitude $\Phi$ should be optimized to have a relative high coverage for all the MEO objects every day. Therefore, the unknown parameter is $\Phi$, and the objective function should be defined as the daily average maximum observation number of the global navigation satellites. It also can be defined as

$$
\min f(\Phi)=N_{\text {total }}-\frac{1}{365} \sum_{n=1}^{365} N_{n}(\Phi)
$$

where $N_{n}$ is the number of global navigation satellites which are observed in the n-th day and $N_{\text {total }}$ is the total number of the global navigation satellites. Therefore, $N_{n}(\Phi)$ is always a nonnegative integer with different $\Phi$, and $f(\Phi)$ has the characteristics of non-continuity and step change. In theory, there is a high coverage rate for global navigation satellites if the observation regions cover almost all the joint regions, and the coverage rate will reduce when observation regions deviate from the joint regions. Therefore, the optimal solution exists in a specific region which makes observation regions cover some of the joint regions. The Hooke-Jeeves algorithm is used to solve this local optimization problem in this paper (Hooke and Jeeves, 1961). In this algorithm, $\sigma$ is the step length for each 
searching, $\beta$ is the reduction factor of step length, and $\xi$ is the end condition of the algorithm. In practice, the observation results will have an obvious change, if the variation of $\Phi$ is at $0.1^{\circ}$ order of magnitude. So, initial parameters are selected as

$$
\Phi_{0}=155^{\circ}, \sigma=3^{\circ}, \beta=0.8, \xi=0.2^{\circ}
$$

It is not necessary to calculate the gradient of the objective function using this algorithm, so that it can find a relative optimal result in a relative short time.

Different sensors with different FOVs have different observation results, and the optimal results are different as well. By optimization, the optimal results of $\Phi$ with different FOVs are listed in Table 2. Observation regions can cover the most of joint regions with $8^{\circ} \mathrm{FOV}$ in the direction of latitude and $65.6^{\circ} \Phi$. While only a part of joint regions can be covered by $6^{\circ}$ or $4^{\circ} \mathrm{FOV}$ in the direction of latitude. The optimization results reveal that with the decline of FOV in the direction of latitude, $\Phi$ is much bigger and sensor prefers to point to joint regions formed by GPS constellation in order to observe more objects. This is because the latitude $\delta$ of joint regions formed by GPS constellation is bigger than that formed by GLONASS constellation. If the sensor points to the joint regions formed by GPS constellation, observation regions can also cover the trajectories of GLONASS satellites. However, if the sensor points to the joint regions formed by GLONASS constellation, it is difficult to observe GPS satellites. Meanwhile, optimization results also reveal that FOV in the direction of latitude plays an important role in observation, while influence of FOV in the direction of longitude to optimization is very small. Similarly, latitude $\delta$ of joint regions formed by Molniya objects is less than that formed by GPS and GLONASS constellations, thus the observation regions can also cover the trajectories of Molniya objects near their apogees with the optimal results of $\Phi$ in Table 2. Moreover, Molniya objects still have relative slow velocity near their apogees, so they can be observed efficiently as well.

\section{Observing results}

The strategy of an equatorial LEO satellite with three sensors payload to observe objects in different fields of sky is proposed in this paper. Two of the sensors are used to observe GEO and GTO objects, and the other sensor is used to observe MEO and Molniya objects. The simulation data of space objects come from the NASA's website (www.space-track.org). And the orbital period of the observation satellite is 13.5 revolutions per day.

\subsection{Observing results of GEO objects}

There are 1022 GEO objects by using a filter for orbital elements $(41000 \mathrm{~km}<R<43000 \mathrm{~km}$, $i \leq 15^{\circ}, e<0.2$ ), including satellites and debris, where $R$ is the semi-major axis. One of the two sensors points along the direction of its position vector in ECI and another one points along the direction of its velocity vector.

Coverage rates for observing GEO objects every day in a whole year under different FOVs are illustrated in Fig. 11. The results reveal that coverage rate for observing GEO objects is mainly influenced by FOV in the direction of latitude $P$. With the increasing of $P$, the more GEO objects can be observed. Meanwhile, coverage rates have decreased during March and September due to the influence of the earth shadow near the vernal equinox and autumnal equinox. The blind zones caused by the earth shadow and the constraint of sun-target-observer angle are distributed symmetrically. Thus, some of the GEO objects with large inclinations, whose ascending nodes exist in one of the blind zones and their descending nodes will also probably exist in another, could not be observed with limited FOV of the sensor.

Observation conditions are similar during the winter and summer, as well as the spring and autumn. So, only the number of observations in a winter and spring day are given, as they are illustrated in Fig. 12. The results reveal that the number of observations in a day for observing GEO objects is mainly influenced by $P$ as well. In order to revisit GEO objects more times in a day, $P$ should be broader. Moreover, the smaller the inclinations of GEO objects, the more easily they can be observed and the more times they can be revisited in a day. Fig. 12 also reveals that most of GEO 
objects can be observed more than 4 times in a summer or a winter day, while fewer observation times of GEO objects observed in a spring or an autumn day for the influence of earth shadow. Meanwhile, there are many GEO objects which could be observed around twenty times. Inclinations of all these objects are less than $0.5^{\circ}$.

FOV in the direction of longitude $Q$ mainly influences the duration of every observation. When $Q$ is $4^{\circ}$, the duration of every observation for GEO objects is about $63 \mathrm{~s}$ on average. When it is $6^{\circ}$ and $8^{\circ}$, the duration increases to about $86 \mathrm{~s}$ and $112 \mathrm{~s}$ respectively. As a result, it is an efficient way to generally observe GEO objects under this strategy for the high revisit frequency and high coverage rate in a day.

\subsection{Observing results of GTO objects}

There are 194 GTO objects by using a filter for orbital elements $(20500 \mathrm{~km}<R<26875 \mathrm{~km}$, $i \leq 15^{\circ}, e>0.6$ ). The strategy is same as that when observing GEO objects. Coverage rates for observing GTO objects every day in a whole year are illustrated in Fig. 13. The number of observations for observing GTO objects in a winter and spring day are illustrated in Fig. 14. According to the results, the conclusion is similar with that when observing GEO objects. The results reveal that coverage rates decline around March and September, which are also caused by the influence of the earth shadow. Moreover, GTO objects, whose apogees exist in the blind zone, are difficult to be observed. So, it is difficult to cover all the GTO objects in a day. The number of observations is mainly related to the inclinations and arguments of perigee of GTO objects. GTO objects would be observed more times if their inclinations are smaller and their apogees are in the effective observation regions.

\subsection{Observing results of MEO objects}

There are 271 MEO objects (mainly consist of global navigation satellites) by using a filter for orbital elements $\left(24000 \mathrm{~km}<R<28000 \mathrm{~km}, 52^{\circ}<i<68^{\circ}, e<0.2\right)$. Coverage rates of different FOVs with different optimal $\Phi$ in Table 2 for observing MEO objects every day in a year are illustrated in Fig. 15 and every two days in a year are illustrated in Fig. 16. The results reveal that with the increasing of $P$, more objects can be observed. Although all the MEO objects couldn't be observed in a day, coverage rates increase with the growth of days. On the other hand, coverage rates are uniform in the whole year. The reason is that sun-target-observer angle are always less than the acceptable value, and objects will not be sheltered from the earth shadow when they are covered by the sensor. The amount of MEO objects observed in different days with different FOVs is listed in Table 3. Almost all the global navigation satellites could be observed within 5 to 6 days using a sensor with $8^{\circ} \mathrm{FOV}$ in the direction of latitude.

Observation results for a specific MEO objects (SSN Number ${ }^{1}$ :40731) with different FOVs are listed in Table 4 . The results reveal that the observation duration is mainly influenced by $Q$, when the orbital period is appointed. The duration exceeds 1 minute per observation, so it is a feasible way to observe MEO objects under this strategy.

\subsection{Observing results of Molniya objects}

There are 114 Molniya objects by using a filter for orbital elements ( $20000 \mathrm{~km}<R<30000 \mathrm{~km}$, $58^{\circ}<i<68^{\circ}, \omega>180^{\circ}, e>0.5$ ), where $\omega$ is the argument of perigee. The strategy is same as that when observing global navigation satellites. Molniya objects are easier to be observed due to their relative slow velocity near their apogees. Similar with observing global navigation satellites, coverage rates are also uniform in the whole year, and mainly influenced by the parameter $P$. Thus, observation results under $4^{\circ} \times 2^{\circ}, 6^{\circ} \times 2^{\circ}$, and $8^{\circ} \times 2^{\circ}$ FOVs are only given, as they are illustrated in Fig. 17. Only a few Molniya objects can't be observed with $6^{\circ} \mathrm{FOV}$ in the direction of latitude. Some of Molniya objects, whose arguments of perigee are close to $270^{\circ}$, can be observed more easily.

Observation results for a specific Molniya object (SSN Number: 40297) with different FOVs are listed in Table 5. The results reveal that the observation duration is also mainly influenced by $Q$, when

\footnotetext{
${ }^{1}$ The number of a space object in the U.S. Space Surveillance Network
} 
the orbital period is appointed. The duration exceeds 70s per observation, so it is a feasible way to observe Molniya object under this strategy.

\section{Conclusions}

In this article, a space-based observation strategy focused on the pointing geometry for beyond-LEO objects using an equatorial LEO satellite carrying multiple sensors is discussed under the relative ideal illumination conditions. GEO, GTO, MEO, and Molniya objects can be observed simultaneously.

The distribution characteristics of GEO, GTO, MEO, and Molniya objects are discussed firstly. According to the characteristics of equiphase distribution for global navigation satellites constellations, joint regions formed by the tracks superposition of two adjacent orbits are proposed for efficient space-based optical observation. As a result, it is necessary to cover different fields of sky where joint regions are distributed in order to observe these objects efficiently. An earth-oriented equatorial LEO satellite is used to perform observation, for its operation characteristics which can make the observation region cover specific fields of sky with specific latitudes.

The strategies for observing GEO, GTO, MEO, and Molniya objects are designed respectively. In order to offset the influence of the earth shadow and the constraint of the sun-target-observer angle, also to increase the number of observations in a day, a satellite with two sensors is proposed to observe GEO and GTO objects. One of the sensors points along the direction of its position vector in ECI, and another point along the direction of its velocity vector. Effective observation regions of the two sensors are complementary, and the blind zone caused by the constraint of the sun-target-observer angle decreases. In order to make the observation region cover the joint regions, the third sensor should point outside of the equatorial plane. By calculation, required installation angles $\Phi$ of the sensor to observe MEO and Molniya objects are similar. Therefore, only using a sensor can observe these objects simultaneously. Meanwhile, accurate values of $\Phi$ with different FOVs are achieved by optimization.

Finally, the capability of an equatorial LEO satellite whose orbital period is 13.5 revolutions per day carrying three sensors with different FOVs is discussed. FOV in the direction of latitude mainly influences the coverage rate and the number of observations, while FOV in the direction of longitude mainly influences the observation duration. On the premise that guaranteeing the observation duration, appropriately increasing the FOV in the direction of latitude can get a better observation effect. With appropriate FOVs, there is a high coverage rate and revisit frequency every day for GEO, GTO and Molniya objects, and a high coverage rete every two days for observing MEO objects. As a result, it's a feasible and efficient way to observe GEO, GTO, MEO, and Molniya objects simultaneously using an equatorial LEO satellite carrying three sensors.

\section{Acknowledgements}

This work was supported by the National Science Foundation of P. R. China under Grant No. 51405499 .

\section{References}

Alexander, P. T., Louis, G. Spatial and Temporal Sampling of Polar Regions from Two-Satellite System on Molniya Orbit, J. Atmos. Ocean. T. 28 (2), 977-992, 2011.

Christopher, J. Evolution of the Global Navigation Satellite System (GNSS), P. IEEE. 96(12), 1902-1917, 2008.

Davis, T. M., Thomas, P. J., Story, D. B. Small satellites for operationally responsive space, in: the 4S Symposium 2016, 30 May - 3 June, Valletta, Malta, 2016.

Flohrer, T., Schildknecht, T., Musci, R. Proposed strategies for optical observations in a future European Space Surveillance network. Adv. Space Res. 41, 1010-1021, 2008. 
Flohrer, T., Krag, H., Klinkrad, H., Schildknecht, T. Feasibility of performing space surveillance tasks with a proposed space-based optical architecture. Adv. Space Res. 47, 1029-1042, 2011.

Hengnian, L., Geostationary Satellite Orbital Analysis and Collocation Strategies, National Defend Industry Press, China, 2010.

Hooke, R., Jeeves, T. A. "Direct Search" Solution of Numerical and Statistical Problems, J. Assoc. Comput. Mach. 8(2), 212-229, 1961.

Kaplan, E., Hegarty, C. Understanding GPS: Principles and Applications, 2nd ed. Norwood, MA: Artech House, 2006.

Klinkrad, H. ESA Space Debris Mitigation Handbook, European Space Agency. Darmstadt, Germany, 2003.

Maskell, P., Oram, P. Sapphire: Canada's answer to space-based surveillance of orbital objects, in: Proceedings of the Advanced Maui Optical and Space Surveillance Technologies Conference, September 16-19, 2008, Wailea Beach, Maui, United States, 2008.

Oswald M., Stabroth S., Wagner A. Satellite-based Solutions for beyond-LEO Space Surveillance, in: Proceeding of the 5th European Conference on Space Debris, 30 March - 2 April, 2009, Darmstadt, Germany, 2009. (ESA SP-672, July 2009).

Revnivykh S. G. GLONASS status, development, and application, in: Proc. 2nd Meeting United Nations Int. Committee Global Navig. Satellite Syst. (ICG), Bangalore, India, Sep. 4-7, 2007.

Sánchez, N., Casal, E. O., Ramos-Lerate, M., Perez, E. M. Space Based Optical Images Within a Space Surveillance System, in: The 59th International Astronautical Congress, Scotland, IAC-08-A6.5.6, 2008 .

Scott, R., Wallace, B., Sale, M., Levesque, M., Thorsteinson, S. Toward Microsatellite Based Space Situational Awareness, in: Proceedings of the Advanced Maui Optical and Space Surveillance Technologies Conference, September 10-13, 2013, Wailea, Maui, Hawaii, 2013.

Sharma, J. Space-Based Visible Space Surveillance Performance, J. Guid. Control Dynam.23 (1), 153-158, 2000.

Sharma, J., Stokes, G. H., Von Braun, C., Zollinger, G., Wiseman, A. J. Toward Operational Space-Based Space Surveillance, Lincoln Lab. J. 13(2), 309-334, 2002.

Silha, J., Schildknecht, T., Hinze, A., Utzmann J., Wagner A., Willemsen P., Teston F., Flohrer T. Capability of Space-Based Space Surveillance System to Detect and Track Objects in GEO, MEO and LEO Orbits, in: 65th International Astronautical Congress, Toronto, Canada, IAC-14-A6.1.1, 2014.

Soop, E. M. Handbook to Geostationary Orbits, Kluwer Academic Publishers, Dordrecht, 1994.

Stokes, G. H., Von Braun, C., Sridharan, R., Harrison, D., Sharma, J. The Spaced-Based Visible Program, Lincoln Lab. J. 11(2), 205-238, 1998.

Utzmann, J., Wagner, A. Space-Based Space Surveillance as Complementary Element in an SSA Architecture, in: European Space Surveillance Conference. Madrid, Spain, 2011.

Utzmann, J., Wagner, A., Silha, J., Schildknecht, T., Willemsen, P., Teston, F., Flohrer, T. Space-Based Space Surveillance and Tracking Demonstrator: Mission and System Design, in: 65th International Astronautical Congress, Toronto, Canada, IAC-14-A6.7.5, 2014.

Xiaoli, W., Xiaogong, H., Gang, W., Huijuan, Z., Chengpan, T. Evaluation of COMPASS ionospheric model in GNSS positioning, Adv. Space Res. 51(2013), 959-968, 2013.

Yi, H., Huayan, S., Jianguang, F., Liang, L. Analysis of the optical scattering characteristics of different types of space targets, Meas. Sci. Technol. 25 (2), 1-10, 2014. 
Yunpeng, H., Kebo, L., Wei, X., Lei, C., Jianyu, H. A Novel Space-Based Observation Strategy for GEO Objects Based on Daily Pointing Adjustment of Multi-Sensors, Adv. Space Res. 47(2016), 505-513, 2016.

Zandbergen, R., Dinwiddy, S., Hahn, J., Breeuwer, E., Blonski, D. Galileo orbit selection, in Proc. Inst. Navig. ION GNSS 2004, Long Beach, CA, Sep. 2004. 
Table 1 List of parameters of joint points formed by GPS, GLONASS, and Molniya objects

Table 2 List of optimal results of $\gamma$ with different FOVs

Table 3 The amount of MEO objects observed by different sensors with different FOVs in different days

Table 4 List of the longest observation durations in a day of a specific MEO object (SSN No. 40731) using different sensors with different FOVs

Table 5 List of the longest observation durations in a day of a specific Molniya object (SSN No. 40297) using different sensors with different FOVs

Fig. 1 Observation strategy of an earth-oriented equatorial LEO satellite with three sensors

Fig. 2 Geometrical relationship of joint points

Fig. 3 Joint points formed by GPS in theory

Fig. 4 Joint points formed by GLONASS in theory

Fig. 5 Joint regions in practice (a: left, formed mainly by GPS; b: right, formed mainly by GLONASS)

Fig. 6 Apogees distribution of Molniya objects

Fig. 7 Observation geometry of equatorial satellites. Satellite's FOV in the direction of latitude in orbit 1 is $P$, and its effective observation region is $p$ in practice. Effective observation region of satellite in orbit 2 which has the same FOV is smaller than that of satellite in orbit 1 . In order to cover the same region, satellite in orbit 2 needs to carry a sensor with broader FOV $P^{\prime}$.

Fig. 8 Coverage cases of GEO objects with different inclinations. Tracks of GEO objects and observation region are projected to the earth surface to illustrate their geometrical relationship.

Fig. 9 Effective observation regions of two sensors with different pointing directions (a: left, observation geometry in theory; b: right, simulation in practice)

Fig. 10 Observation geometry of observing global navigation satellites using equatorial satellites

Fig. 11 Coverage rate every day of observing GEO objects using different sensors with different FOVs

Fig. 12 The number of observations for observing GEO objects using different sensors with different FOVs in a winter and spring day

Fig. 13 Coverage rate every day of observing GTO objects using different sensors with different FOVs

Fig. 14 The number of observations for observing GTO objects using different sensors with different FOVs in a winter and spring day

Fig. 15 Coverage rate every day of observing MEO objects using different sensors with different FOVs Fig. 16 Coverage rate every two days of observing MEO objects using different sensors with different FOVs

Fig. 17 Coverage rate of observing Molniya objects (a: above, within every day in a whole year; b: below, within every two days in a whole year) 


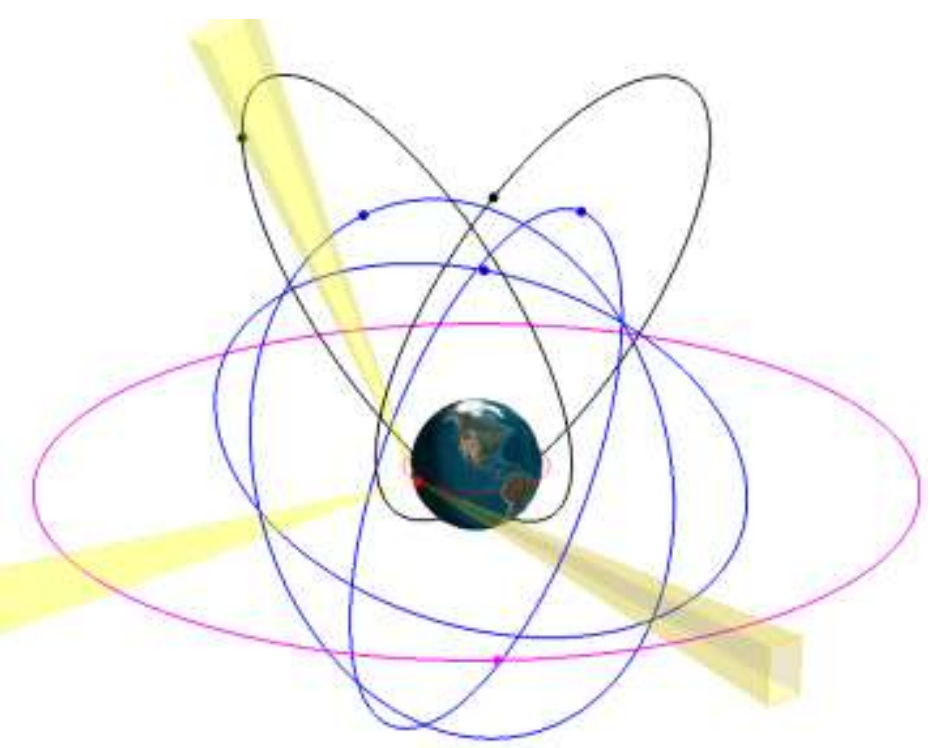

Fig. 1 Observation strategy of an earth-oriented equatorial LEO satellite with three sensors

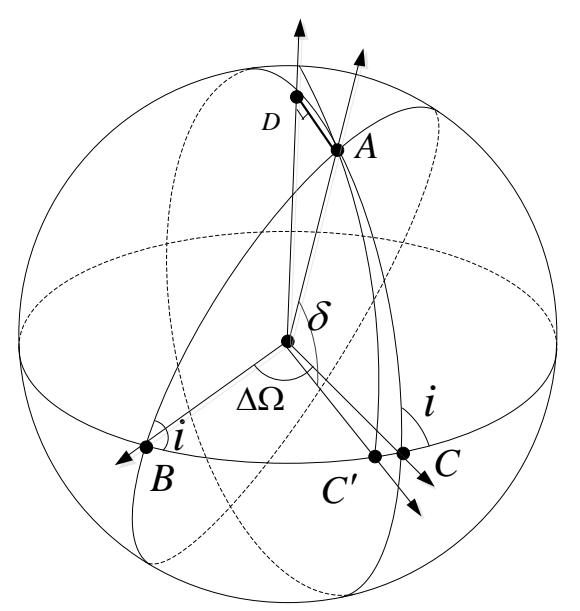

Fig. 2 Geometrical relationship of joint points
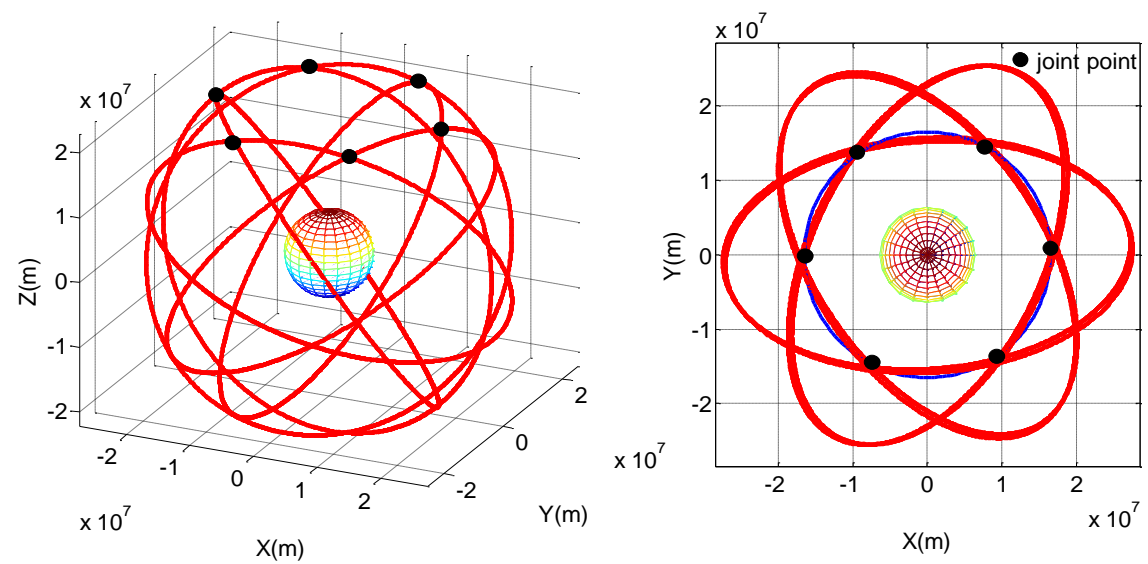

Fig. 3 Joint points formed by GPS in theory 

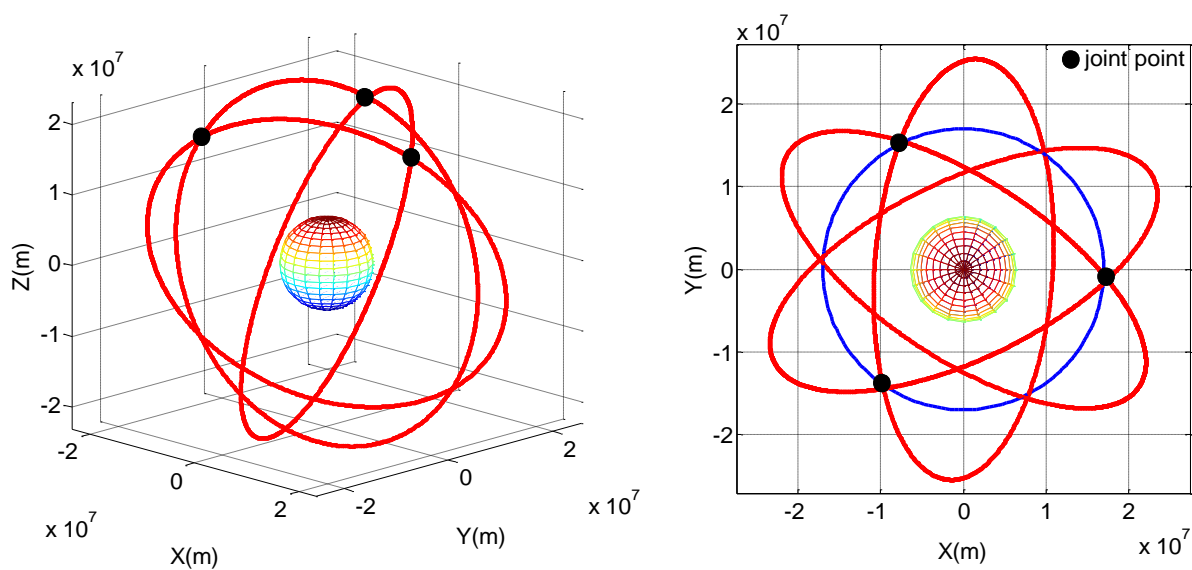

Fig. 4 Joint points formed by GLONASS in theory
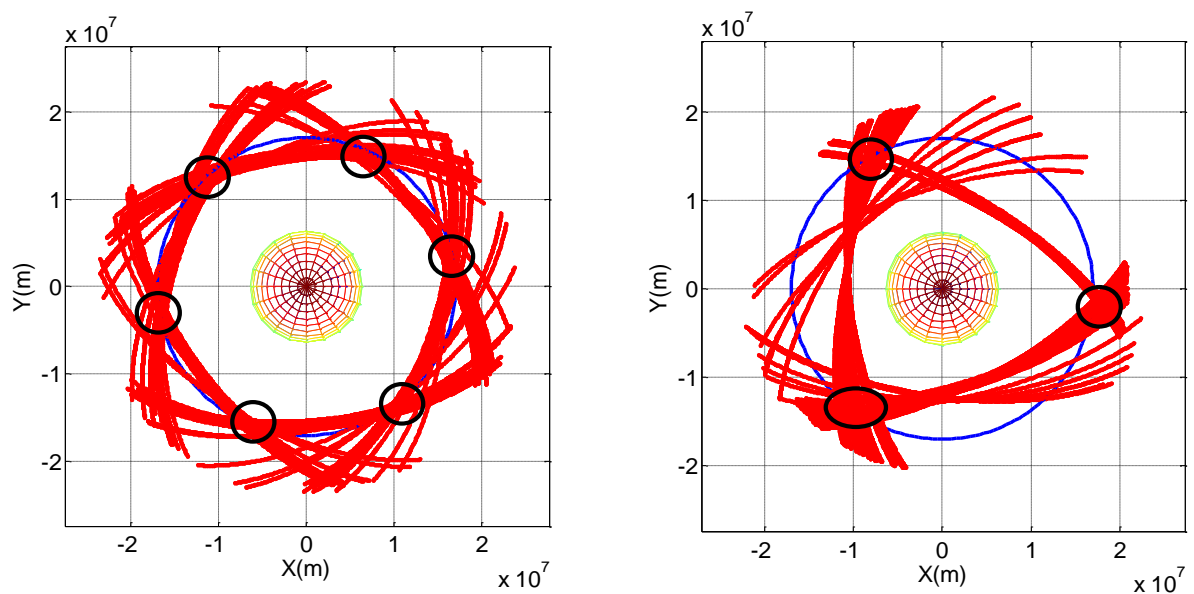

Fig. 5 Joint regions in practice (a: left, formed mainly by GPS; b: right, formed mainly by GLONASS)

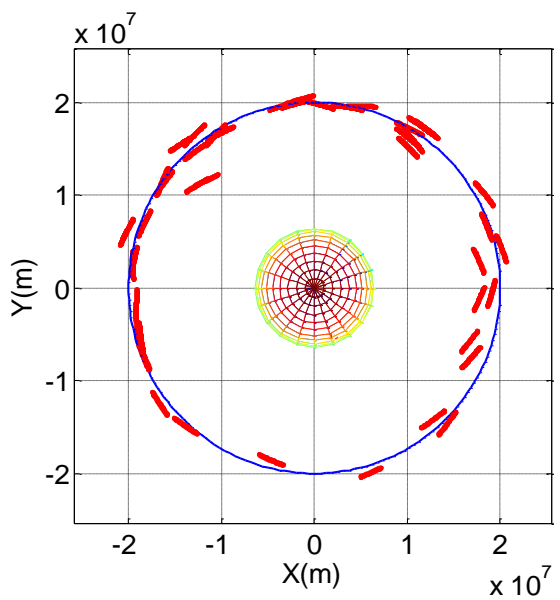

Fig. 6 Apogees distribution of Molniya objects 


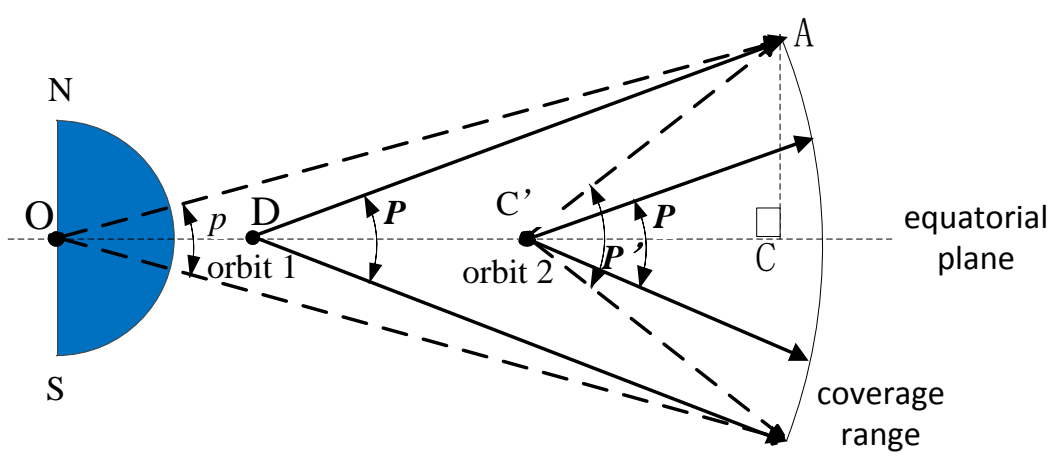

Fig. 7 Observation geometry of equatorial satellites. Satellite's FOV in the direction of latitude in orbit 1 is $P$, and its effective observation region is $p$ in practice. Effective observation region of satellite in orbit 2 which has the same FOV is smaller than that of satellite in orbit 1 . In order to cover the same region, satellite in orbit 2 needs to carry a sensor with broader FOV $P^{\prime}$.

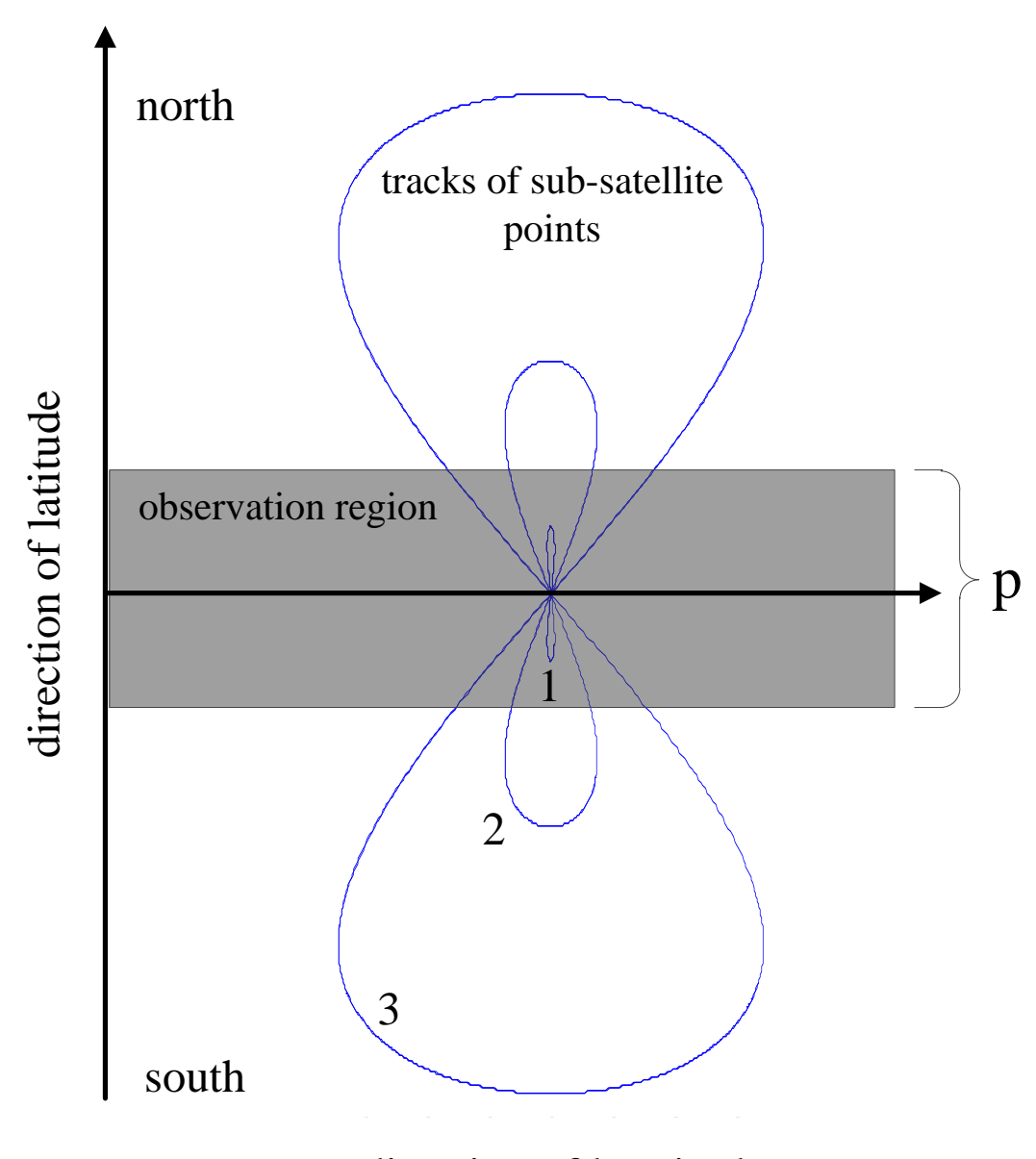

\section{direction of longitude}

Fig. 8 Coverage cases of GEO objects with different inclinations. Trajectories of GEO objects and observation region are projected to the earth surface to illustrate their geometrical relationship. 

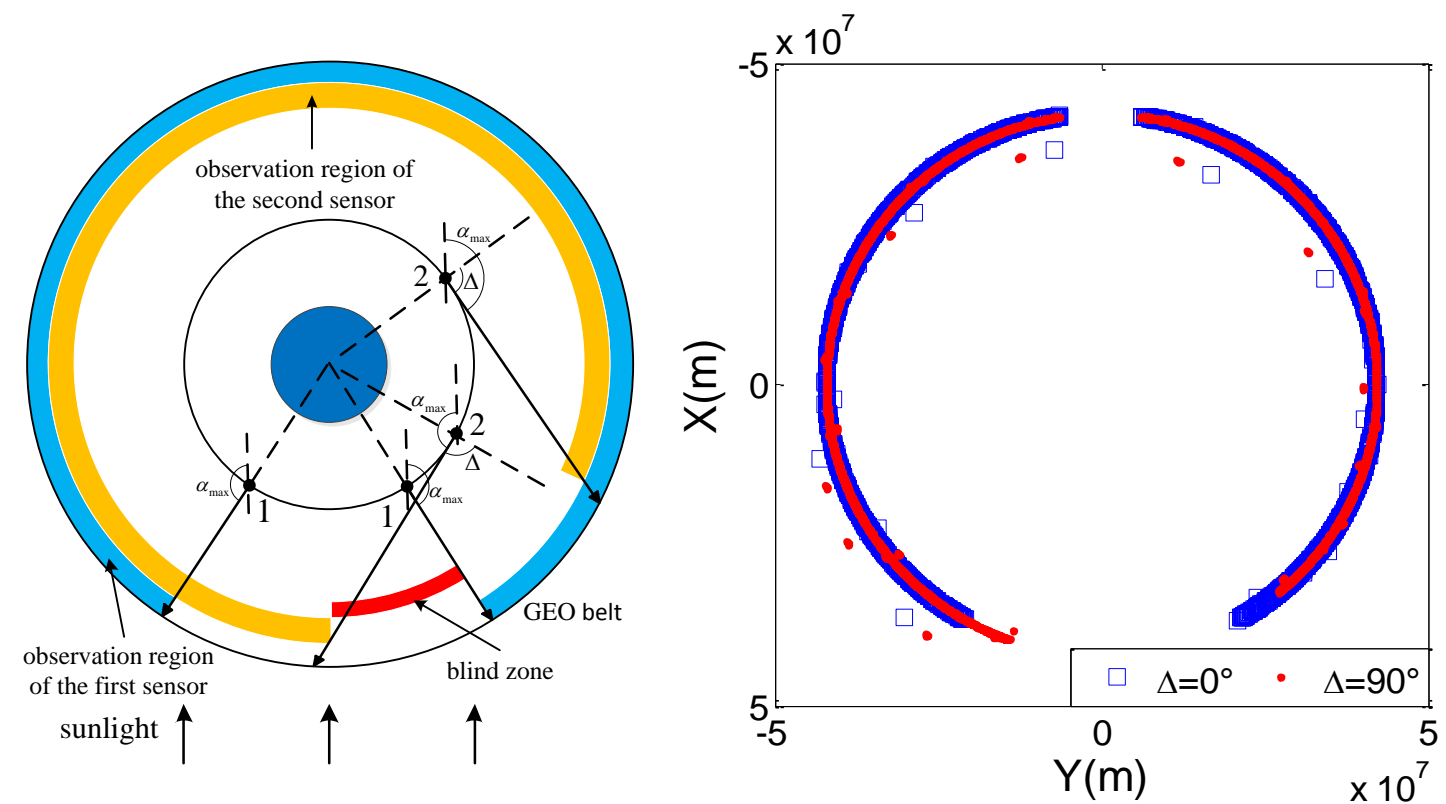

Fig. 9 Effective observation regions of two sensors with different pointing directions (a: left, observation geometry in theory; b: right, simulation in practice)

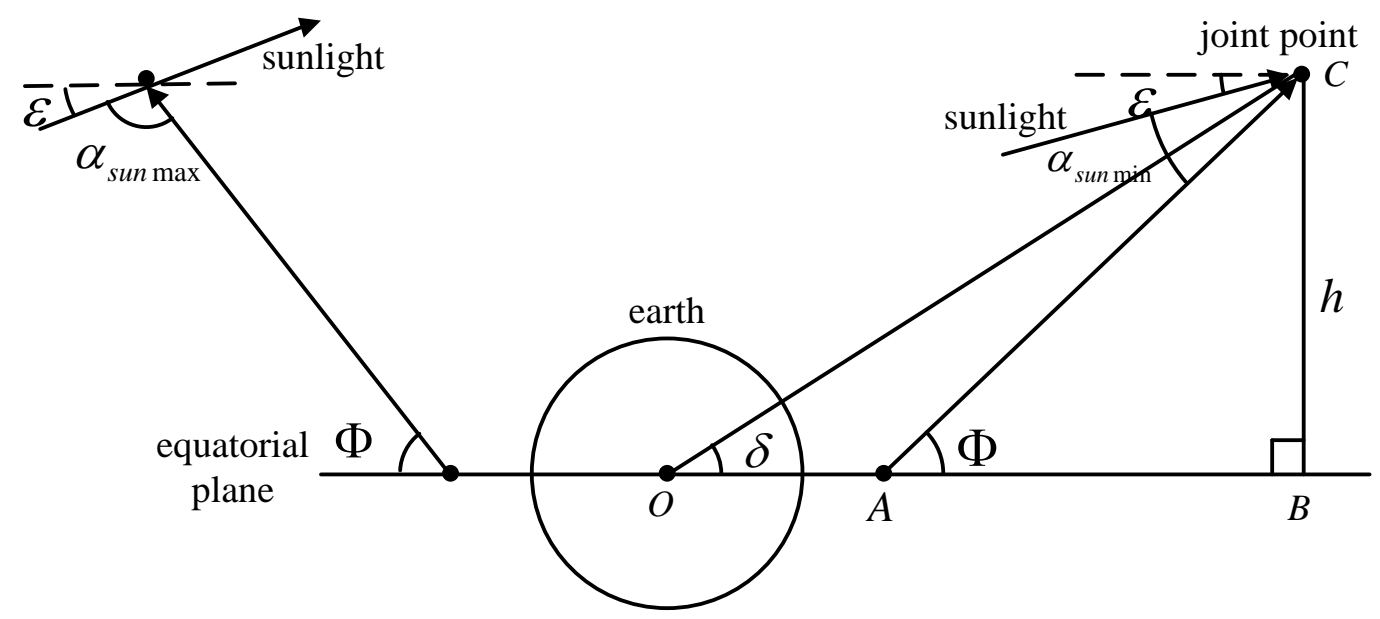

Fig. 10 Observation geometry of observing MEO objects using equatorial satellites 


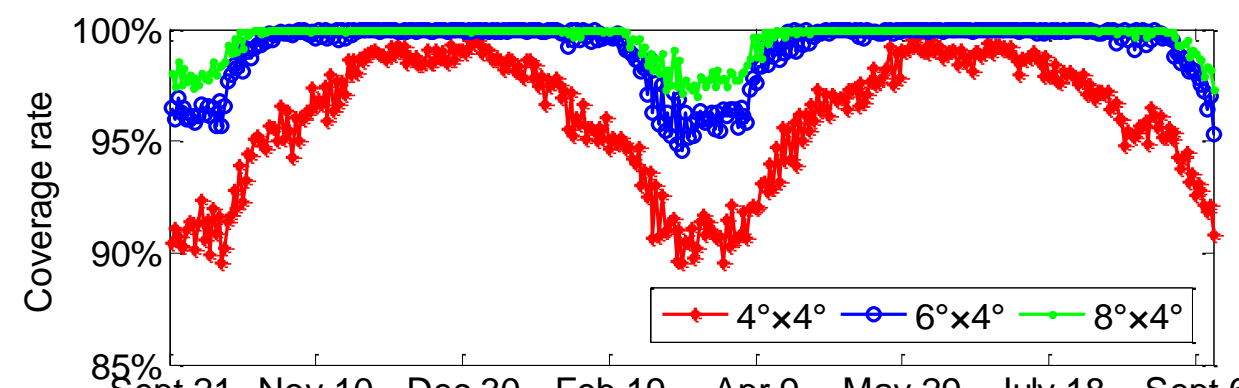

$85 \%$ Sept.21 Nov.10 Dec.30 Feb.19 Apr.9 May.29 July.18 Sept.6

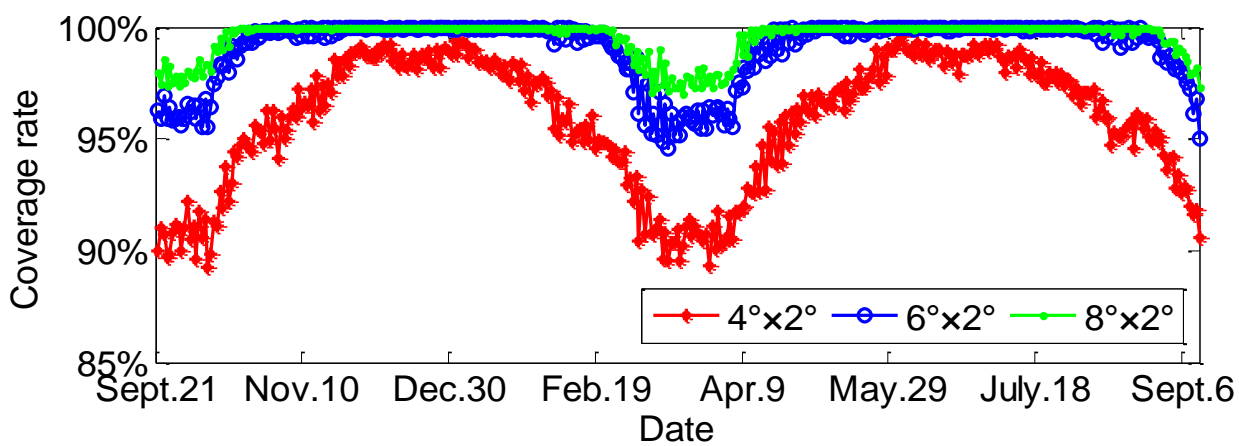

Fig. 11 Coverage rate every day of observing GEO objects using different sensors with different FOVs
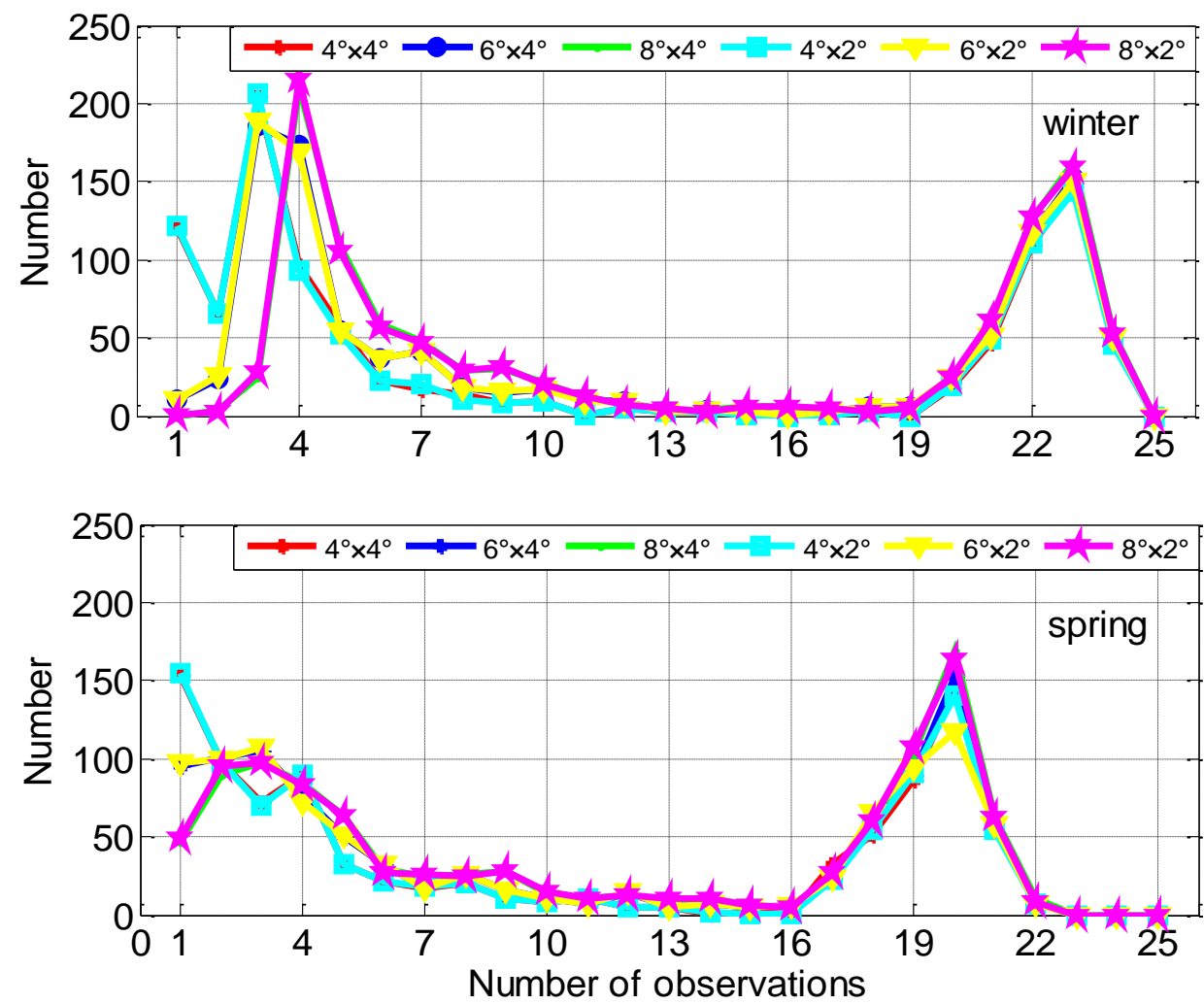

Fig. 12 The number of observations for observing GEO objects using different sensors with different FOVs in a winter and spring day 

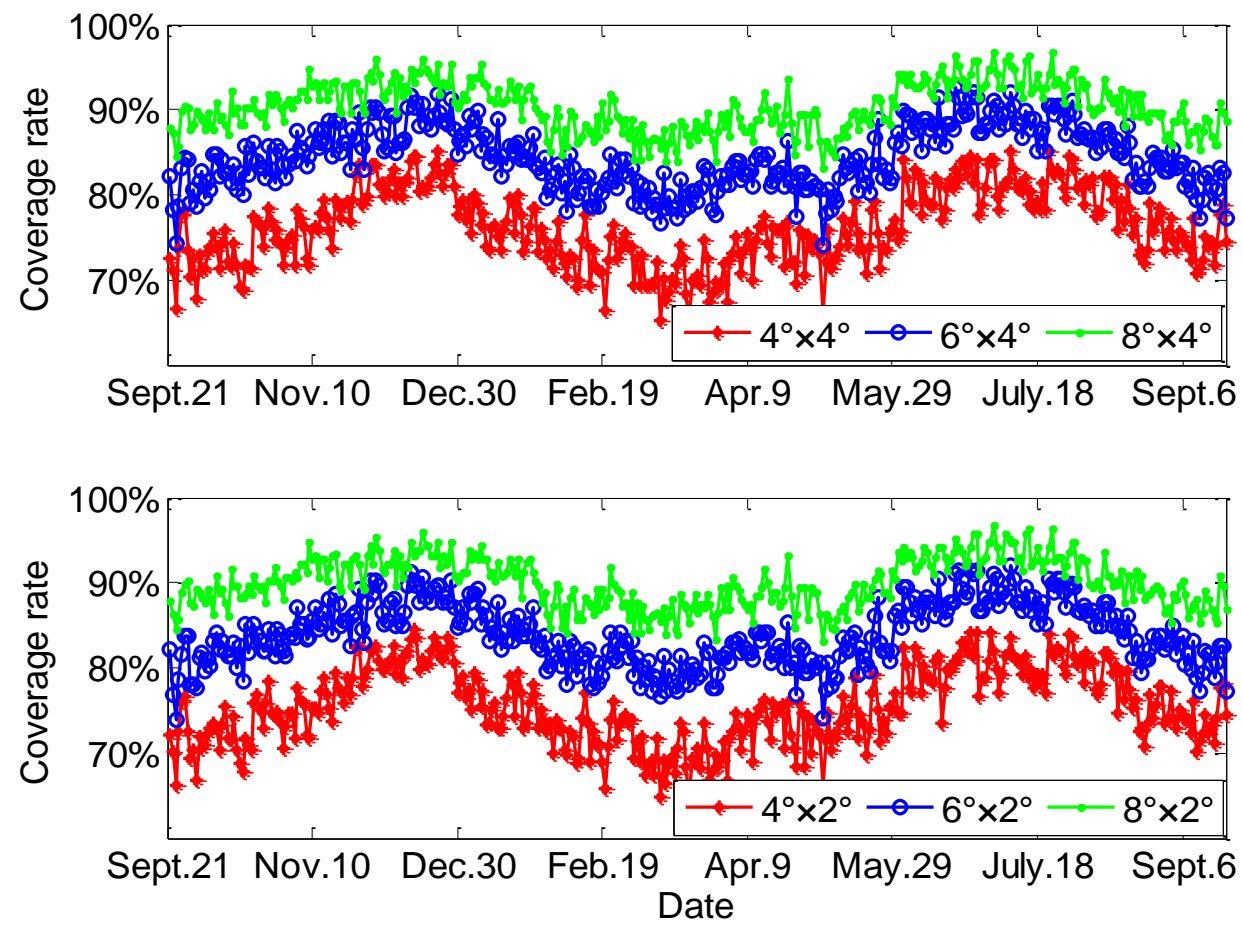

Fig. 13 Coverage rate every day of observing GTO objects using different sensors with different FOVs
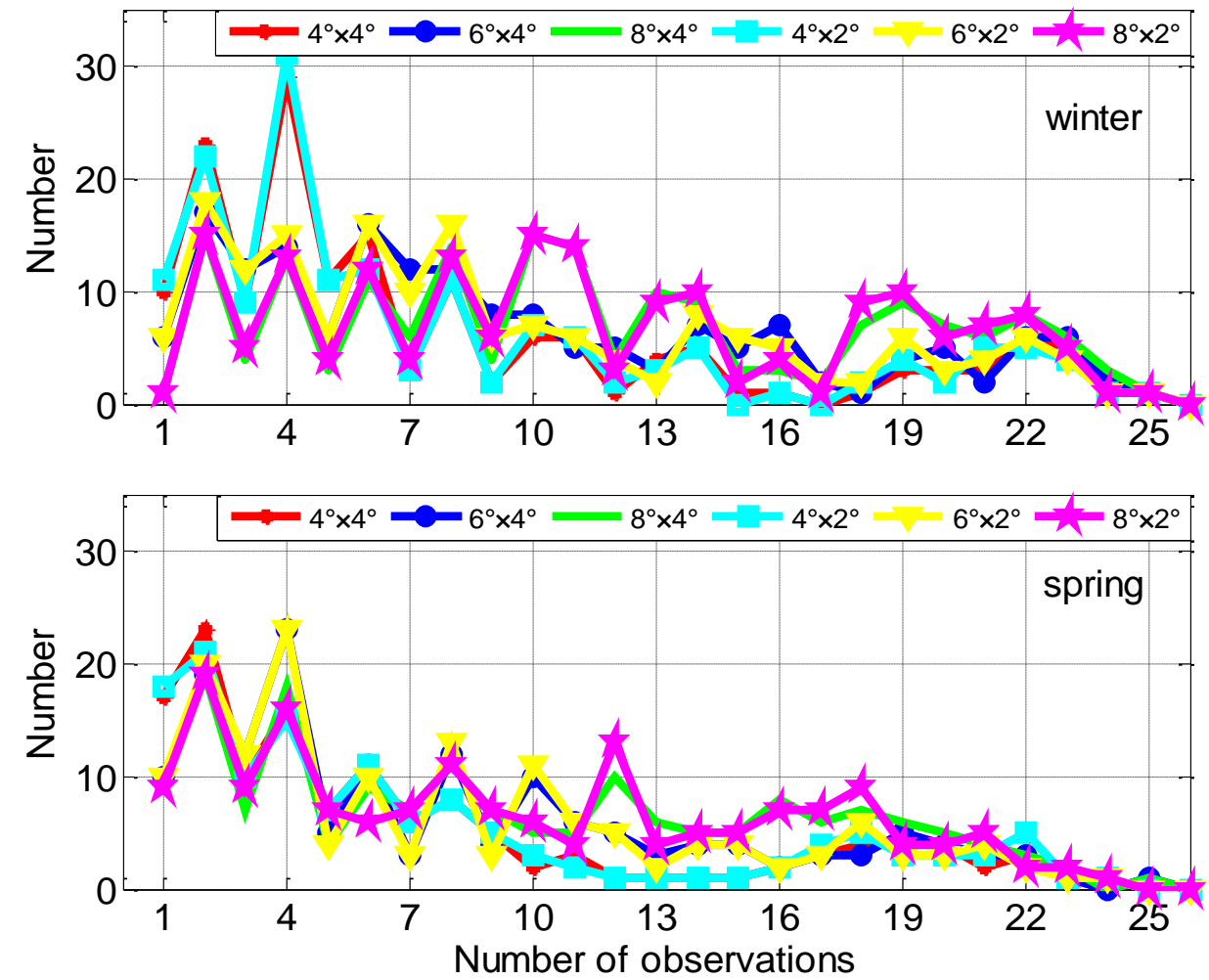

Fig. 14 The number of observations for observing GTO objects using different sensors with different FOVs in a winter and spring day 

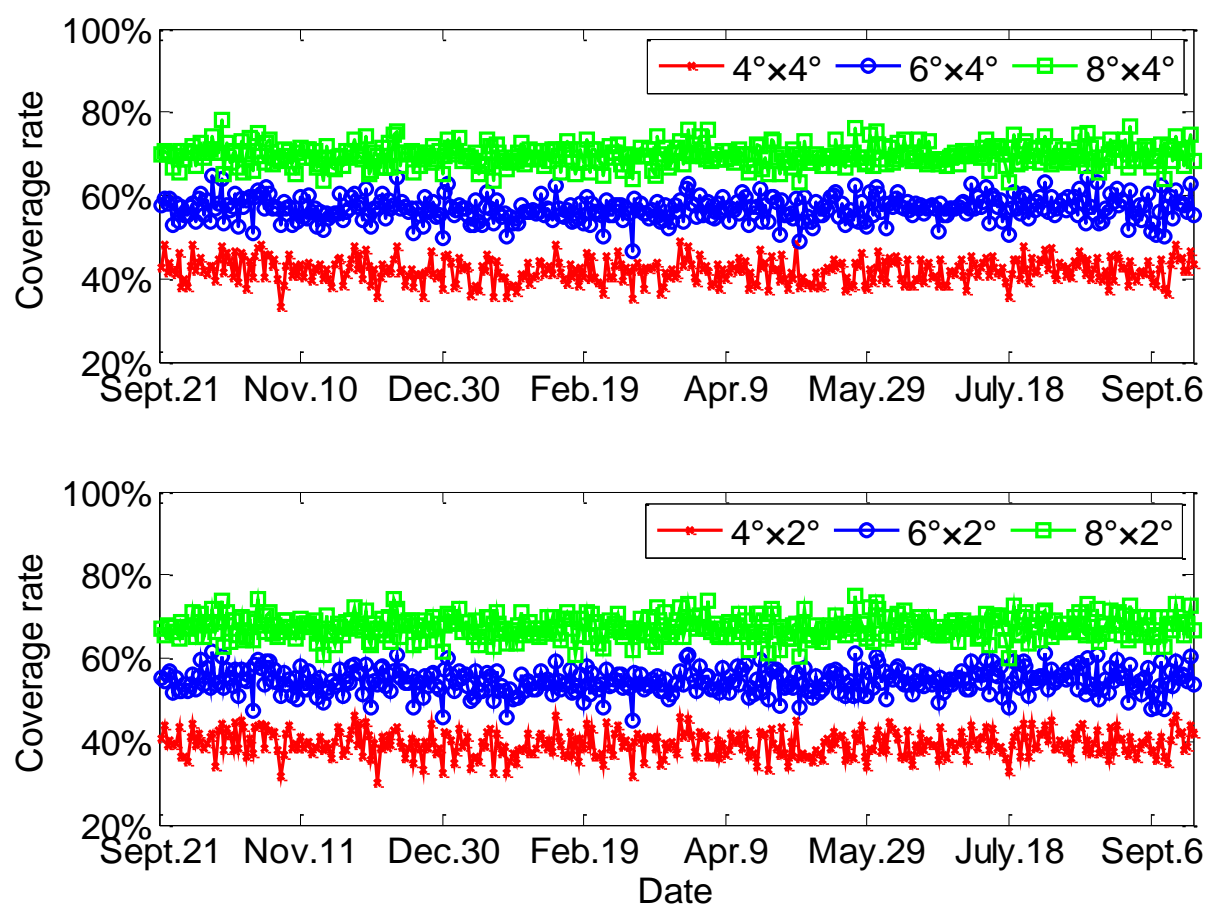

Fig. 15 Coverage rate every day of observing MEO objects using different sensors with different FOVs
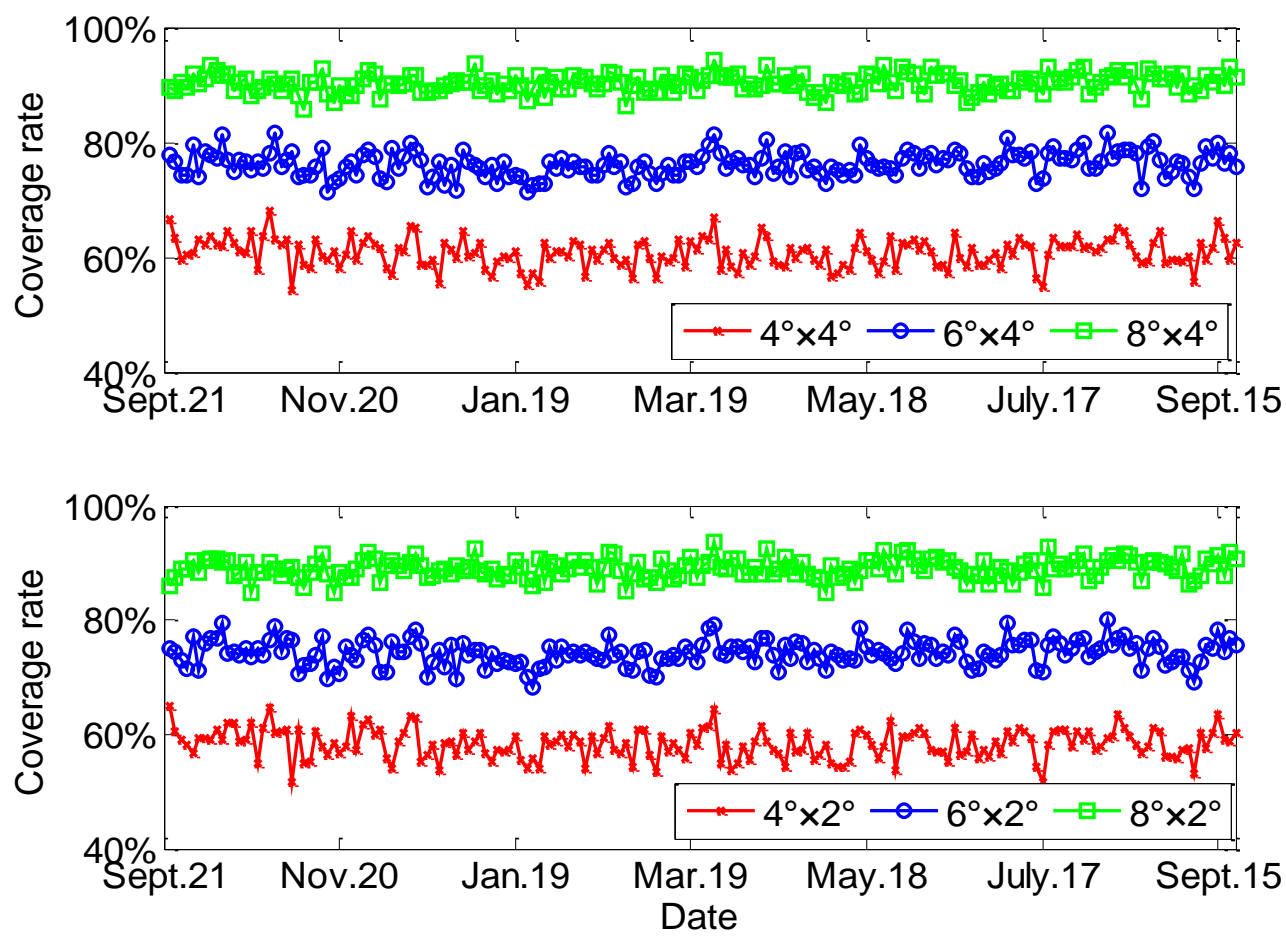

Fig. 16 Coverage rate every two days of observing MEO objects using different sensors with different FOVs 

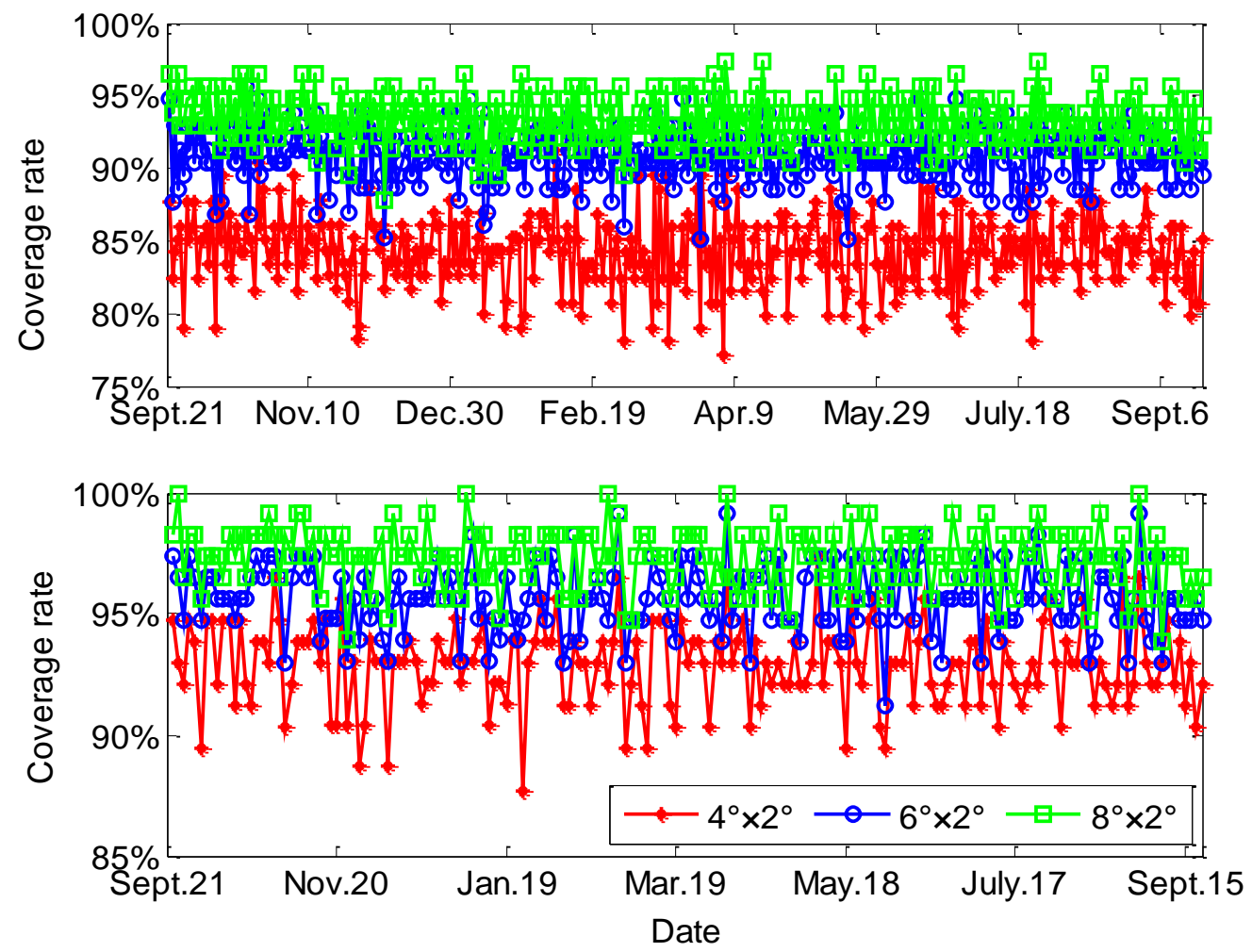

Fig. 17 Coverage rate of observing Molniya objects (a: above, within every day in a whole year; b: below, within every two days in a whole year) 
Table 1. List of parameters of joint points formed by GPS, GLONASS, and Molniya objects

\begin{tabular}{cccc}
\hline Objects & $r(\mathrm{~km})$ & $h(\mathrm{~km})$ & $\delta\left(^{\circ}\right)$ \\
\hline GPS & 16699 & 20653 & 51.1 \\
GLONASS & 17461 & 18554 & 46.7 \\
Molniya & 20498 & 40933 & 63.4 \\
\hline
\end{tabular}

Table 2. List of optimal results of $\Phi$ with different FOVs

\begin{tabular}{ccccccc}
\hline FOV & $8^{\circ} \times 4^{\circ}$ & $6^{\circ} \times 4^{\circ}$ & $4^{\circ} \times 4^{\circ}$ & $8^{\circ} \times 2^{\circ}$ & $6^{\circ} \times 2^{\circ}$ & $4^{\circ} \times 2^{\circ}$ \\
\hline$\Phi$ & $65.6^{\circ}$ & $66.6^{\circ}$ & $67.4^{\circ}$ & $65.5^{\circ}$ & $66.5^{\circ}$ & $67.4^{\circ}$ \\
\hline
\end{tabular}

Table 3. The amount of MEO objects observed by different sensors with different FOVs in different days

\begin{tabular}{crrrrrr}
\hline FOV & $8^{\circ} \times 4^{\circ}$ & $6^{\circ} \times 4^{\circ}$ & $4^{\circ} \times 4^{\circ}$ & $8^{\circ} \times 2^{\circ}$ & $6^{\circ} \times 2^{\circ}$ & $4^{\circ} \times 2^{\circ}$ \\
\hline 3 days & 261 & 221 & 189 & 255 & 216 & 185 \\
4 days & 263 & 236 & 199 & 259 & 233 & 195 \\
5 days & 267 & 245 & 211 & 264 & 242 & 210 \\
6 days & 267 & 252 & 225 & 265 & 248 & 221 \\
\hline
\end{tabular}

Table 4. List of the longest observation durations in a day of a MEO object (SSN No. 40731) using different sensors with different FOVs

\begin{tabular}{ccccccc}
\hline FOV & $8^{\circ} \times 4^{\circ}$ & $6^{\circ} \times 4^{\circ}$ & $4^{\circ} \times 4^{\circ}$ & $8^{\circ} \times 2^{\circ}$ & $6^{\circ} \times 2^{\circ}$ & $4^{\circ} \times 2^{\circ}$ \\
\hline Longest duration & $124 \mathrm{~s}$ & $124 \mathrm{~s}$ & $124 \mathrm{~s}$ & $64 \mathrm{~s}$ & $64 \mathrm{~s}$ & $64 \mathrm{~s}$ \\
\hline
\end{tabular}

Table 5. List of the longest observation durations in a day of a specific Molniya object (SSN No. 40297) using different sensors with different FOVs

\begin{tabular}{ccccccc}
\hline FOV & $8^{\circ} \times 4^{\circ}$ & $6^{\circ} \times 4^{\circ}$ & $4^{\circ} \times 4^{\circ}$ & $8^{\circ} \times 2^{\circ}$ & $6^{\circ} \times 2^{\circ}$ & $4^{\circ} \times 2^{\circ}$ \\
\hline Longest duration & $135 \mathrm{~s}$ & $135 \mathrm{~s}$ & $135 \mathrm{~s}$ & $71 \mathrm{~s}$ & $71 \mathrm{~s}$ & $70 \mathrm{~s}$ \\
\hline
\end{tabular}

